\title{
The Association Between Dietary Acid Load and Odds of Migraine: A Case-Control Survey
}

\author{
Maryam Mousavi · Soodeh Razeghi Jahromi • Mansoureh Togha • \\ Zeinab Ghorbani · Azita Hekmatdoost • Pegah Rafiee • \\ Boshra Torkan · Pedram Shirani · Hossein Ansari · Ahmadreza Karami • \\ Morvarid Nourmohammadi · Faezeh Khorsha
}

Received: January 6, 2021 / Accepted: March 24, 2021 / Published online: April 24, 2021

(C) The Author(s) 2021

\section{ABSTRACT}

Introduction: It has been shown that a high acid load in the human body can affect inflammatory factors and the nitric oxide pathway. These factors are also thought to play

Maryam Mousavi and SoodehRazeghi Jahromi contributed equally to this work.

Supplementary Information The online version contains supplementary material available at https:// doi.org/10.1007/s40120-021-00247-2.

M. Mousavi · S. R. Jahromi · A. Hekmatdoost . P. Rafiee · B. Torkan · P. Shirani · A. Karami · M. Nourmohammadi Department of Clinical Nutrition and Dietetics, Faculty of Nutrition and Food Technology, Shahid Beheshti University of Medical Sciences, Tehran, Iran

M. Mousavi · S. R. Jahromi · M. Togha ( $₫)$. P. Rafiee - B. Torkan · P. Shirani - A. Karami . M. Nourmohammadi · F. Khorsha Headache Department, Iranian Centre of Neurological Research, Neuroscience Institute, Tehran University of Medical Sciences, Tehran, Iran e-mail: toghae@sina.tums.ac.ir

\section{Z. Ghorbani · F. Khorsha}

Department of Cardiology, Cardiovascular Diseases Research Centre, School of Medicine, Heshmat Hospital-Guilan University of Medical Sciences, Rasht, Iran

H. Ansari

Department of Neurology, University of California San Diego (UCSD), San Diego, USA an important role in the initiation of migraine attacks. We have therefore explored the association between dietary acid load and odds of migraine in a case-control study.

Methods: The migraine group $(n=514$, diagnosed according to the International Classification of Headache Disorders 3rd edition [ICHDIII] criteria) was recruited from a tertiary headache clinic. The controls consisted of 582 sex-matched healthy volunteers who were randomly selected from the general population. A validated 168-item semiquantitative food frequency questionnaire was used for dietary intake assessments. Estimation of the dietary acid load was performed using three different measures: potential renal acid load (PRAL) score, net endogenous acid production (NEAP) score, and protein/potassium ratio.

Results: Multivariable logistic regression analysis showed that, in comparison with the lowest tertile, the highest tertile of dietary acid load measures, including PRAL (odds ratio [OR] 7.208, 95\% confidence intervals [CI] 3.33-15.55), and NEAP (OR 4.108, 95\% CI 1.924-8.774) scores and the protein/potassium ratio (OR 4.127, 95\% CI 1.933-8.814), significantly increased the odds of migraine ( $P$ value for trend $\leq 0.001$ ).

Conclusion: In this study, high dietary acid load was associated with higher odds of migraine. It is therefore possible that restricting dietary acid load could reduce the odds of migraine in susceptible subjects. However, due 
to the preliminary nature of the current evidence, caution is advised in drawing a definitive conclusion. More well-designed studies are required for a better understanding of this important issue.

Keywords: Dietary acid load; Headache; Inflammation; Migraine; Nitric oxide

\section{Key Summary Points}

\section{Why carry out this study?}

Migraine is one of the most disabling neurological issues worldwide and has been associated to other serious health problems, such as coronary heart disease.

High acid load in the human body is associated with inflammatory factors and the nitric oxide pathway. These factors are also thought to play an important role in the initiation of migraine attacks.

The aim of this study was to evaluate the association between dietary acid load and odds of migraines in a case-control study.

\section{What was learned from the study?}

Subjects with the highest levels of dietary acid load were shown to have significantly higher odds of migraine disease in comparison to lowest levels.

As a strategy to reduce dietary acid load, it is possible that restricting the intake of acidogenic food may be associated with a reduction in the odds of migraine.

\section{DIGITAL FEATURES}

This article is published with digital features, including a summary slide, to facilitate understanding of the article. To view digital features for this article go to https://doi.org/10.6084/ m9.figshare.14269373.

\section{INTRODUCTION}

Migraine ranks as one of the most disabling neurological diseases, affecting one in seven people worldwide [1]. According to the latest report of the Global Burden of Diseases, Injuries and Risk factors (GBD) study, the estimated prevalence of headache disorders was about 3 billion of the general population in 2016 [2]. Migraine has been estimated to be responsible for 45.12 million years of life lived with disability, most prevalently in women aged between 15 and 49 years [3]. It has also been related to other health problems, such as anxiety, depression, fibromyalgia, asthma, obesity, stroke, and coronary heart disease [4, 5]. Although the underlying mechanisms for the onset of migraines are not entirely realized, several abnormal conditions in the body are involved in the pathophysiology of this disorder. Some of these mechanisms include changes in cerebral blood flow, mitochondrial and hormonal dysfunction, genetic factors, obesity, neuroinflammation, in addition to increased levels of some neuropeptides, especially nitric oxide (NO) and calcitonin gene-related peptide (CGRP). Activation of the trigeminovascular system could lead to the excretion of substances such as CGRP, substance P, and NO [6-9]. These agents are associated with inflammation and vasodilation in cranial vessels, which lead to intense pain $[6,7,9]$. NO is believed to play an important role in initiating migraine attacks through activation of the $\mathrm{NO} /$ cyclic guanosine monophosphate (cGMP) pathway. There is also evidence that NO levels are elevated in people with migraine during and between headache attacks [10, 11]. Moreover, cytokines and inflammatory agents around nerves can activate trigeminovascular neurons and increase CGRP release [12]. It has been shown that the levels of inflammatory factors, such as tumor necrosis factor-alpha (TNF $\alpha)$, C-reactive protein, and interleukin 6 , increase in people with migraine headache compared to healthy subjects [11, 13-16].

Available evidence suggests that even a slight increase in acid load may stimulate the expression of induced NO synthases, increase levels of 
inflammatory factors, such as TNF $\alpha$, and increase blood flow and adiposity measures (measurements that are used for obesity, such as body mass index [BMI], waist circumference, among others) [17-21]. High dietary acid load can be identified by both a high intake of acid precursors (including phosphorus and proteins containing high levels of sulfur-containing amino acids) and a low intake of base precursors (such as potassium, magnesium, and calcium). Chronic consumption of diets with high acid load could shift the acid-base balance towards acidosis [22, 23]. This type of diet is rich in meat, dairy and grain and low in fruits and vegetables [23]. A previous case-control study [24] addressed the hypothesis that the Western diet could increase odds of migraine disease as it is typically characterized by a heavy consumption of meat and low consumption of vegetables and fruit, potentially representing a high dietary acid load [25].

A high acid load in the human body could be associated with inflammation, NO pathways, blood flow, and adiposity measures [17-21]. These factors are also thought to play a role in the initiation of migraine attacks $[6,7,9,11,13-16]$. Thus, it can be hypothesized that there is relationship between dietary acid load and the development of migraine in susceptible subjects. The aim of the study reported here, therefore, was to explore the association between dietary acid load and the odds of migraine headaches.

\section{METHODS}

\section{Patients and Procedures}

In the current case-control study, migraineurs were recruited from the tertiary headache clinic of Sina University Hospital and a private headache clinic. The data were collected from 2015 to 2018. Detailed information on the study methods has been reported previously [26]. Our expert headache-specialist neurologist diagnosed migraine headache (episodic and chronic migraine) according to the International Classification of Headache Disorders 3rd edition (ICHDIII) (ICHDIII criteria, beta version) [27].
Because patients with episodic migraine are less likely to be referred to our tertiary headache clinic, a higher proportion of referred patients were diagnosed with chronic migraine. Based on the ICHDIII-beta criteria, chronic migraine is defined as $\geq 15$ days of headache per month with at least eight migraine-type headaches in the last 3 months, and episodic migraine is characterized as up to 14 days of headache per month. Controls consisted of 582 sex-matched healthy volunteers who were randomly selected from the general population. Inclusion criteria for both the patients and the control group included: age between 18-60 years, BMI between 18.5 and $35 \mathrm{~kg} / \mathrm{m}^{2}$, and daily energy intake between 800 and $5000 \mathrm{kcal}$. Exclusion criteria for both groups were: pregnancy or breastfeeding; a history of chronic diseases, such as gastrointestinal, liver, or kidney disorders; diabetes mellitus; cardiovascular diseases; malignancy; tuberculosis; sarcoidosis; rheumatoid arthritis; psychiatric disorders; any neurological diseases other than migraine, including Parkinson's, Alzheimer's disease, multiple sclerosis, tension-type headache, among others; substance/alcohol overuse or consistent smoking; and following a specific diet over the last year.

The patients referred to the tertiary headache clinic were prescribed medications by our expert headache-specialist neurologist. These medicines comprised: drugs for abortive treatment of acute headache attack (i.e., nonsteroidal anti-inflammatory drugs, other analgesics [codeine], and triptans), prophylactic medications (i.e., propranolol [beta-blockers], topiramate and sodium valproate/Depakene [antiepileptic drugs]), selective serotonin reuptake inhibitors, and tricyclic antidepressants).

Written informed consent was obtained from all study subjects. The research methods and study design were reviewed and approved by the ethics committee of the vice-chancellor of research affairs (ethics code: IR.TUMS.NI.REC.1398.010). The study was conducted in accordance with the Helsinki Declaration of 1964 and its later amendments. 


\section{Assessment of Anthropometric Measures}

For anthropometric variables, body weight was measured using a Seca 755 medical scale (weighing accuracy: $0.5 \mathrm{~kg}$; Seca $\mathrm{GmbH} \& \mathrm{Co}$. KG, Hamburg, Germany), and height was measured with a standard stadiometer (accuracy: $0.1 \mathrm{~cm})$. BMI was calculated as weight $(\mathrm{kg})$ divided by the square of the height $\left(\mathrm{m}^{2}\right)$.

\section{Dietary Assessment and Calculation of Dietary Acid Load}

Food intake was assessed using a validated 168-item semiquantitative food frequency questionnaire (FFQ) [28]. Trained dietitians who were blinded to the study group completed the FFQ for all participants. Food items were appraised according to the US Department of Agriculture standard serving sizes. Household measures (e.g., beans, 1 tablespoon; chicken meat, 1 leg or wing; rice, 1 large or small plate) were employed when standard serving sizes did not fit [29]. Participants reported the intake of each food item on the FFQ based on daily, weekly, monthly, or yearly intake. The intake data were then converted to grams assuming each month consisted of 30.5 days. Daily macronutrient intake for each individual was determined using the National Food Composition Table of Iran [30]. Total macronutrient consumption was measured by summing up macronutrient intake of all consumed foods [31].

In this study, we used three different measures for calculating the dietary acid load, namely, potential renal acid load (PRAL), net endogenous acid production (NEAP), and protein/potassium ratio; all three measures have been validated in previous studies [32, 33]. The algorithms of these three measures are as follows: (1) PRAL $(\mathrm{mEq} /$ day $)=(0.49 \times$ protein $(\mathrm{g} /$ day $))+(0.037 \times$ phosphorus $\quad(\mathrm{mg} /$ day $))$ $-(0.021 \times$ potassium $\quad(\mathrm{mg} /$ day $))-(0.026 \times$ magnesium $\quad(\mathrm{mg} /$ day $))-(0.013 \times$ calcium $(\mathrm{mg} /$ day $))$ [32]; (2) NEAP $(\mathrm{mEq} /$ day $)=(54.5 \times$ protein intake $[\mathrm{g} /$ day $] \div$ potassium intake [mEq/day]) - 10.2 [25]; and (3) protein/ potassium ratio $=$ total protein/potassium intake (both g/day) [33].

\section{Statistical Method}

Analyses were performed applying the Statistical Package for the Social Sciences (SPSS) software version 24 (SPSS Inc./IBM Corp., Armonk, NY, USA). For demographic factors, we compared the BMI, nutrient intake, and food groups between the case and control groups using the independent sample $t$ test and Chi-squared test. All dietary variables that were investigated regarding the odds of migraine were classified in tertiles. Accordingly, baseline characteristics, anthropometric data, and nutritional intakes were compared through the PRAL and NEAP tertiles. One-way analysis of variance and the Chi-squared test were used to compare continuous and categorical variables between PRAL and NEAP tertiles, respectively. Data were reported as the mean \pm standard deviation (SD) or number with the percentage, as appropriate. To explore the association between dietary acid load measures and odds of migraine, adjusted odds ratios (ORs) and 95\% confidence intervals (CIs) were obtained using multivariable logistic regression models following adjustment for age (year, continuous), sex, BMI $\left(\mathrm{kg} / \mathrm{m}^{2}\right.$, continuous), total daily energy intake (kcal/day, continuous) as well as daily intake of carbohydrates (g/day), total fat (g/day), $\mathrm{Na} \mathrm{(mg/day),} \mathrm{and}$ dietary food groups (i.e., total refined grains, whole grains, total sugar, vegetables, fruits, red meat, poultry and egg, processed meat, fish, dairy, and nuts). In order to test for linear trends across tertiles, the median value of each tertile of dietary acid load measures was considered to be a continuous variable. All reported $P$ values were two-sided, and a $P$ value of $<0.05$ was considered to indicate statistical significance.

\section{RESULTS}

In total, 1096 participants (approx. 94\% were women) were assessed in this study, of whom 514 were migraine patients with a mean (SD) age of 36.21 (9.79) years and 582 were healthy with a mean age of 44.85 (13.85) years. The 
mean (SD) BMI in migraine patients and healthy subjects was 25.91 (4.75) and 28.12 (4.80) $\mathrm{kg} / \mathrm{m}^{2}$, respectively. Of the 514 patients with migraine, 279 had chronic migraine and 235 had episodic migraine. Demographic factors, BMI, nutrient intake, and food group details between the case and control groups are shown in Table 1. Generally, compared to those subjects without migraine, those with migraine disease were found to have a significantly higher consumption of total animal protein, total fat, dairy, processed meat, poultry, eggs, and fruits and also a lower consumption of total plant protein, grains, and vegetables.

Individuals in the third tertile of PRAL (median score 13.06) and NEAP (median score 71.99) scores were younger and tended to have a higher intake of refined grains, sugar, processed meats, total protein, and total fat and a lower intake of vegetables, fruits, nuts, dairy, total energy, total carbohydrate, plant protein, and sodium than those in the first tertile of PRAL (median score - 27.05) and NEAP (median score 31.11$)$ scores ( $P$ values $<0.05$ ). Mean intake of fish, poultry, and eggs was similar across the PRAL and NEAP tertiles ( $P$ values $>0.05$ ) (Table 2). Also, regarding PRAL scores, individuals in the second tertile (median score - 1.53) had a higher BMI and a higher intake of red meat and animal protein than those in the first tertile (median score - 27.05) $(P$ values $<0.05)$. For NEAP scores, subjects in the third tertile (median score 71.99) and the second tertile (median score 49.80) had a greater consumption of whole grains than those in the first tertile (median score 31.11) $(P$ value $=0.005)($ Table 2$)$.

The ORs and corresponding 95\% CIs for migraine according to tertiles of dietary acid load measures of intake are shown in Table 3. According to multivariable logistic regression analysis, in comparison with the lowest tertile, individuals in the third tertile of PRAL scores had an increased odds of migraine by approximately sevenfold (OR 7.208, 95\% CI 3.33-15.55; $P$ for trend $<0.001)$. Also, subjects in the third tertile of each NEAP score or protein/potassium ratio were shown to have an approximately fourfold increased odds of migraine when compared to those in the first and second tertiles (OR 4.10, 95\% CI 1.928.77; $P$ for trend $<0.001$; and OR $4.12,95 \%$ CI $1.93-8.81 ; P$ for trend $<0.001$, respectively) (Table 3).

Additionally, there was no significant association between chronic and episodic migraine with regard to dietary acid load measures such as PRAL, NEAP, and the protein/potassium ratio (Electronic Supplementary Material).

\section{DISCUSSION}

The result of this large population-based observational study showed that high dietary acid load intake had a direct association with odds of migraine onset. Higher PRAL scores are associated with a high daily intake of protein and phosphorus (as acid-producing components) and a lower daily intake of potassium, magnesium, and calcium (as base-producing components) [32, 34, 36]. In our study, participants with higher PRAL scores had approximately a sevenfold increased odds of migraine onset compared to those with the lowest scores. Also, higher NEAP scores and a higher protein/ potassium ratio, indicating a higher daily consumption of protein divided by potassium $[25,33]$, were related to about a fourfold elevation in the odds of migraine onset.

This investigation used three different methods for calculating the dietary acid load: PRAL, NEAP, and the protein/potassium ratio. These methods are based on protein, potassium, phosphorus, calcium, and magnesium intake, which are acid-base precursors and likely related to $\mathrm{pH}$ homeostasis in the human body $[23,32,33]$. Higher PRAL and NEAP scores and higher protein/potassium ratios indicate the intake of more acidic foods, while lower values for these measurements suggest the consumption of more alkaline food items. Individuals with a high dietary acid load have been found to have a lower intake of fruits and vegetables and a higher consumption of meat and protein consumption [23]. In our study, we found that high PRAL and NEAP scores and high protein/potassium ratio, which are related to meat, grain, and sugar consumption, were positively associated with the odds of migraine. 
Table 1 Comparison of cases and controls with a regard to demographic factors, body mass index, nutrient intake and food groups

\begin{tabular}{|c|c|c|c|}
\hline Variables & Case $(n=514)$ & Control $(n=582)$ & $P$ value ${ }^{\mathrm{a}}$ \\
\hline Chronic migraine (\%) & 54.28 & - & - \\
\hline Episodic migraine (\%) & 45.71 & - & - \\
\hline Female (\%) & $485(94.4 \%)$ & $550(94.5 \%)$ & 0.91 \\
\hline Age (years) & $36.21 \pm 9.79$ & $44.85 \pm 13.85$ & $<0.001^{*}$ \\
\hline Body mass index $\left(\mathrm{kg} / \mathrm{m}^{2}\right)$ & $25.91 \pm 4.75$ & $28.12 \pm 4.80$ & $<0.001^{*}$ \\
\hline \multicolumn{4}{|l|}{ Nutrient intake } \\
\hline Total energy (kcal) & $2201.78 \pm 691.33$ & $2175.24 \pm 550.89$ & 0.480 \\
\hline Total carbohydrate (g) & $240.69 \pm 114.20$ & $236.58 \pm 71.82$ & 0.471 \\
\hline Total protein $(\mathrm{g})$ & $89.77 \pm 24.03$ & $92.10 \pm 26.33$ & 0.127 \\
\hline Total animal protein $(\mathrm{g})$ & $42.58 \pm 15.42$ & $37.34 \pm 15.08$ & $<0.001^{*}$ \\
\hline Total plant protein $(\mathrm{g})$ & $33.69 \pm 12.73$ & $39.71 \pm 13.69$ & $<0.001^{*}$ \\
\hline Total fat $(\mathrm{g})$ & $114.88 \pm 118.78$ & $84.74 \pm 32.50$ & $<0.001^{*}$ \\
\hline $\mathrm{Na}(\mathrm{mg} /$ day $)$ & $1564.89 \pm 655.93$ & $1644.94 \pm 910.24$ & 0.099 \\
\hline \multicolumn{4}{|l|}{ Food groups } \\
\hline Whole grains (g/day) & $35.00 \pm 47.47$ & $47.44 \pm 67.02$ & $<0.001^{*}$ \\
\hline Refined grains (g/day) & $327.30 \pm 172.03$ & $392.54 \pm 169.21$ & $<0.001^{*}$ \\
\hline Total sugar (g/day) & $69.11 \pm 106.95$ & $59.03 \pm 67.48$ & 0.059 \\
\hline Vegetables (g/day) & $311.62 \pm 178.74$ & $388.13 \pm 199.19$ & $<0.001^{*}$ \\
\hline Fruit (g/day) & $774.21 \pm 835.23$ & $372.94 \pm 181.36$ & $<0.001^{*}$ \\
\hline Nuts(g/day) & $11.97 \pm 19.11$ & $10.87 \pm 16.12$ & 0.300 \\
\hline Dairy (g/day) & $346.78 \pm 201.34$ & $300.43 \pm 178.16$ & $<0.001^{*}$ \\
\hline Red meat (g/day) & $29.95 \pm 25.95$ & $28.73 \pm 21.03$ & 0.390 \\
\hline Processed meat (g/day) & $9.60 \pm 14.48$ & $3.98 \pm 7.27$ & $<0.001^{*}$ \\
\hline Fish (g/day) & $9.51 \pm 9.66$ & $9.08 \pm 11.71$ & 0.502 \\
\hline Poultry and eggs (g/day) & $59.42 \pm 33.43$ & $48.17 \pm 32.19$ & $<0.001^{*}$ \\
\hline
\end{tabular}

Values in table are presented as the mean \pm standard deviation (SD) or frequency (number) with the percentage in parentheses, as appropriate for the variable

*Significant difference at $P<0.05$ between the case and control groups

a $P$ value according to $t$ test or Chi-squared test

Therefore, we conclude that these findings demonstrate that a high acid load in the human body, possibly induced by the intake of acidic food [22], may make an essential contribution to migraine disorder.

To our knowledge, the association between the dietary acid load and odds of migraine has 


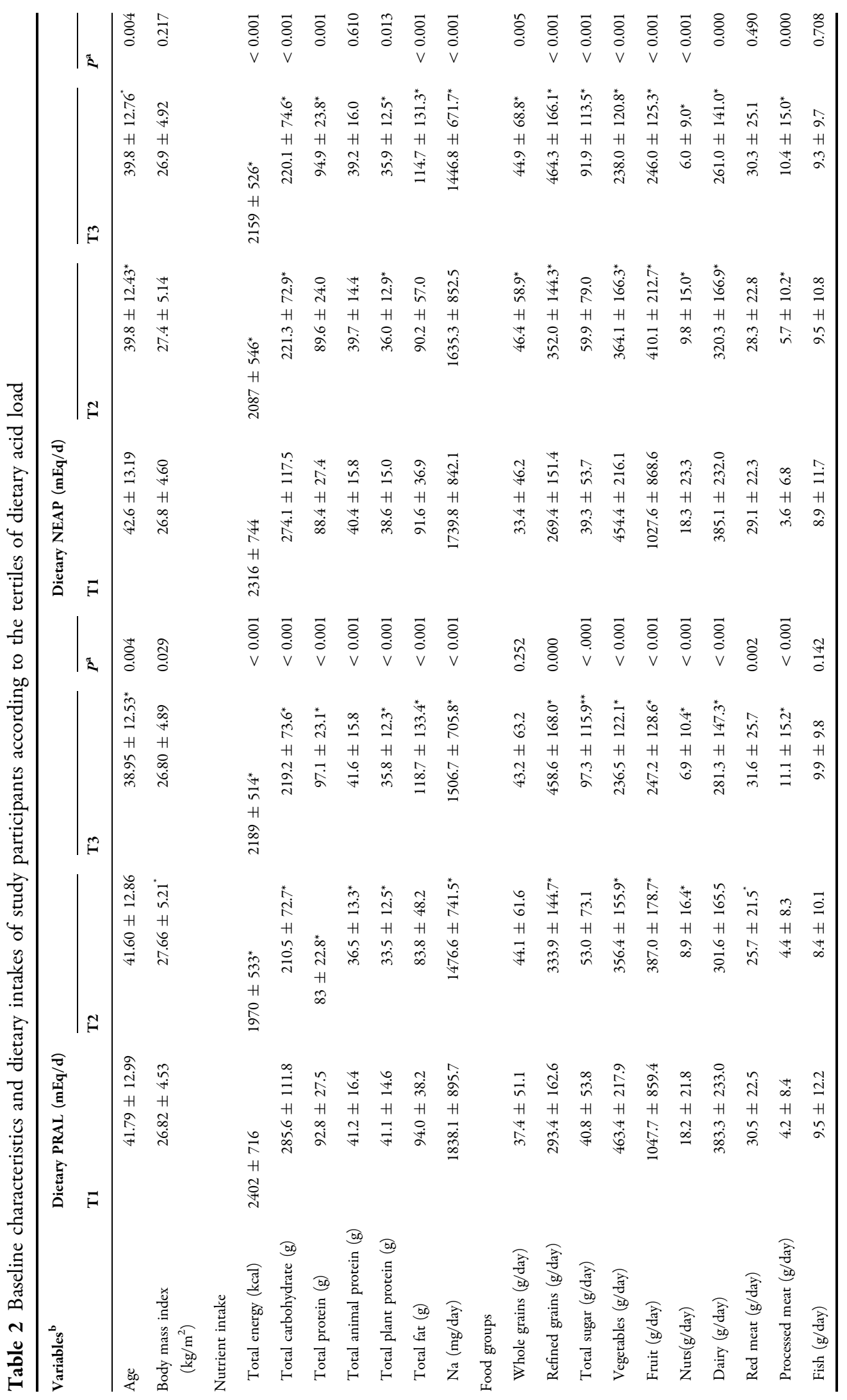




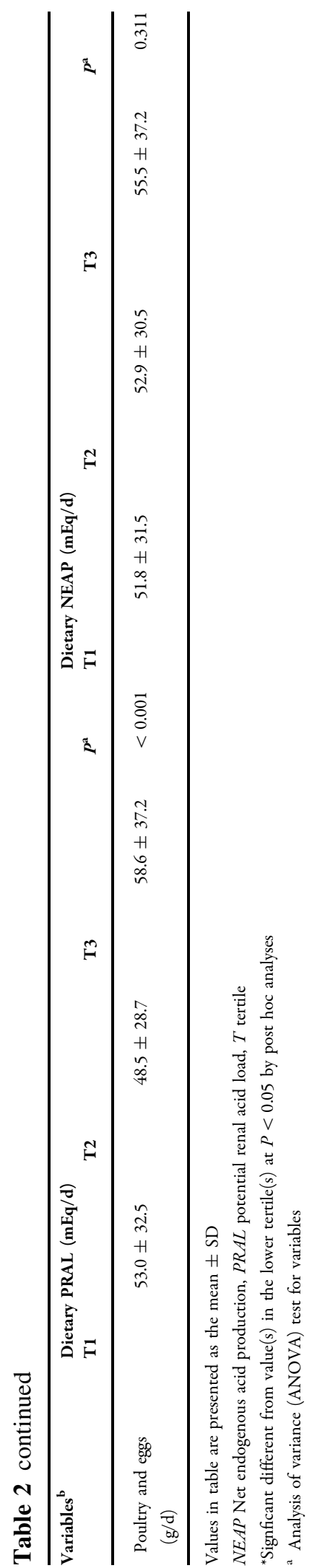

not been studied to date. Previous studies have only reported the consumption of single acidic and basic food items in association with the odds of migraine [36, 37]. In agreement with our findings, one case-control study showed that the odds of migraine in children decreased by about 50 and $70 \%$ with increased consumption of vegetables and fruits, respectively [36]. Another study found that individuals with migraine showed a significantly higher frequency to eat red meat than did individuals without migraine [37]. In addition, according to available evidence, individuals with Western dietary patterns have a higher frequency of migraine attacks [24]. There is a considerable quantity of acidic food items in Western diets, including a high intake of red and processed meat and a low intake of fruits and vegetables. As such, the Western diet may be an example of a diet with a high acid load [25], which is in line with our findings.

It should be noted that chronic consumption of a diet with a high acid load could shift the acid-base balance toward acidosis [22]. For this reason, the balance between consumption of acidic and alkaline food items is probably more important than the exclusive consumption of single acidic and alkaline food items, as has been reported in previous studies. For example, our subjects with migraine disease generally consumed more fruits (alkaline food item) than did the healthy subjects (Table 1). However, the patients also consumed more animal proteins and processed meat (acidic food items) and lower quantities of plant proteins and vegetables (alkaline food items) than the control group. Therefore, it would appear that the participants with migraine disease consumed a diet that was unbalanced in terms of acidic and basic food items. Of note, as no previous study has examined the effects of an acid-base balance in the diets of migraine patients, this potential explanation needs further study.

Although protein intake can influence the acid-base balance in the human body due to sulfuric amino acid content [23], a recent study revealed no differences in dietary protein intake among women with and without migraine [38]. In the present study, we found that high PRAL and NEAP values and a high protein/potassium 
Table 3 Odds ratio and $95 \%$ confidence interval for migraine according to the tertiles of dietary acid load

\begin{tabular}{|c|c|c|c|c|}
\hline \multirow[t]{2}{*}{ Indexes of dietary acid load } & \multicolumn{3}{|c|}{ Odds ratio of dietary indexes of acid load ( $95 \%$ confidence interval) } & \multirow[t]{2}{*}{$P$ for trend } \\
\hline & T1 & T2 & T3 & \\
\hline \multicolumn{5}{|l|}{ PRAL (mEq/day) } \\
\hline Cases/non-cases & $197 / 169$ & $138 / 227$ & $179 / 186$ & \\
\hline Multivariable model $^{a}$ & 1.00 & $2.190(1.242-3.861)$ & $7.208(3.339-15.558)$ & $<0.001$ \\
\hline \multicolumn{5}{|l|}{$\mathrm{NEAP}(\mathrm{mEq} /$ day $)$} \\
\hline Cases/non-cases & $207 / 158$ & $142 / 224$ & $165 / 200$ & \\
\hline Multivariable model $^{\mathrm{a}}$ & 1.00 & $1.574(0.910-2.722)$ & $4.108(1.924-8.774)$ & $<0.001$ \\
\hline \multicolumn{5}{|l|}{ Protein/potassium ratio } \\
\hline Cases/non-cases & $207 / 158$ & $141 / 224$ & $166 / 200$ & \\
\hline Multivariable model $^{\mathrm{a}}$ & 1.00 & $1.573(0.910-2.720)$ & $4.127(1.933-8.814)$ & $<0.001$ \\
\hline
\end{tabular}

ratio, which is significantly associated with high total protein intake, were positively associated with the odds of migraine. The observed difference between the results of our study and those of the earlier study may be related to the dietary patterns and dietary habits of the respective study populations as well as various controlled confounders. Another explanation for these differences could be that in our study the dietary acid load measures (PRAL, NEAP, and protein/potassium ratio) were calculated based on the balance between the intake of acid and base precursors. Therefore, it is possible that total protein intake, as an acid precursor, in combination with the intake of base precursors, has different effects on the odds of migraine that does total protein consumption alone.

It should be mentioned that migraine disease may alter dietary preferences in these patients. For this reason, there is likely to be a two-sided relationship between food intake and migraine disorder. However, it is not clear if migraine can cause high dietary acid load or not. Therefore, more causative studies are needed to identify the exact association.

Chronic consumption of a diet with a high acid load could shift the acid-base balance toward acidosis [22]. The dietary acid load is also more likely to induce acidosis when there are additional abnormalities in the acid-base balance in the human body [19]. It has been found that people with migraine may have abnormalities in their acid-base balance due to mitochondrial dysfunction [39, 40]. Several possible mechanisms have been proposed to account for for the association between high dietary acid load and the onset of migraine attacks or increasing headache attack frequency in migraineurs [17-21].

First, animal and human studies have reported that acidosis can cause high levels of inflammation and NO due to its damaging effect on tissues and/or blood vessels $[17-19,41]$. In the physiological context, TNF $\alpha$, myeloperoxidase, and NO synthases may be produced under acidosis conditions in the human body [19]. Inflammation and factors such as CGRP and NO are believed to play significant roles in initiating migraine attacks $[42,44]$ while cytokines and inflammatory agents around nerves could activate trigeminovascular neurons and increase CGRP release by cellular mechanisms. Due to the majority of $\mathrm{TNF} \alpha$ receptors being in trigeminal ganglion neurons, TNFa may increase CGRP gene expression in trigeminal ganglion neurons by 
increasing the expression of intracellular mitogen-activated protein (MAP) kinase signaling pathways, which in turn may be a triggering factor in the onset of headache [43-47]. Additionally, NO can cause intense pain by increasing the dilation of and inflammation in cranial vessels through the NO-cGMP pathway $[10,11]$. Therefore, acidosis may be positively associated with the initiation of migraine attacks by augmenting inflammation and NO synthesis.

Second, a high acid load might cause an increase in cortisol level. High cortisol levels can have a negative impact on pain recovery in migraineurs [48]. Also, elevated cortisol levels, which may be augmented by acidosis, appear to be associated with high blood flow and BMI in the human body $[20,21]$. It has been reported that high blood flow and BMI may play negative roles in the onset of migraine pathogenesis. For example, the odds of migraine has been found to increase in obese individuals [16, 49]. Moreover, although evidence on the association between hypertension and migraineis is controversial, several findings have been reported indicating that long duration and uncontrolled hypertension, especially high systolic blood pressure, may be positively linked to migraine with/without aura [50, 51].

Third, gut microbiota could be influenced by dietary acid load due to low intake of fruits and vegetables. Thus, microbial diversity may be reduced in gastrointestinal systems and, in turn, lead to adverse effects on human microbiomes [52]. Recent shreds of evidence suggest the existence of a link between gut microbiota and migraine [8]. Studies have also reported potential beneficial effects following probiotic supplementation in subjects with migraine headache $[16,53,54]$.

Taken together, there appears to be evidence showing that a high acid load may contribute to heightened odds of migraine by increasing the inflammatory state; elevating cortisol levels; modifying the NO signaling pathway, blood flow, and gut-microbiota; and affecting body weight and hypertension risk [17-21, 41, 48, 52]. However, the design of the present study does not allow us to determine the causal relationship between high dietary acid load and odds of migraine disorder. For example, as mentioned, it is also possible turn the situation around, i.e., that having a migraine may alter dietary preferences. Additionally, no previous research has investigated the association between dietary acid load and odds of migraine. For these reasons, more welldesigned studies are required for a better understanding of this important issue. The mechanisms discussed above may provide the direction for future research examining this potential association.

There are several strengths to our study. First, dietary acid load was assessed by three methods, namely, PRAL, NEAP, and protein/potassium ratios, which were estimated using a validated FFQ. Second, the case group comprised a relatively large population of migraineurs, all of whom were diagnosed by our expert neurologist-headache specialist according to the ICHDIII beta criteria. Third, to our knowledge, this was the first study to explore the association between dietary acid load measures and migraine odds.

However, there are also a number of limitations that need to be considered. First, the exact dosages of medications used and the frequency and intensity of migraine headache attacks were not considered in the calculations. Second, body acid-base balance assessments were based on dietary calculations. Had urinary and serum $\mathrm{pH}$ measurements also been available, the results would be more accurate. Third, most of the enrolled participants in the present study were women, possibly because women suffer from migraine approximately by two- to threefold more than men. As such, this type of headache is often recognized in women while a higher proportion of men with migraine remains underdiagnosed; this may be associated with suboptimal treatment in men and thus a lower participation of men in the related studies [55]. Fourth, the control group was drawn randomly from the general population, matched for gender with the migraine group, but we did not match the cases and controls according to their age; in our study, the subjects in the control group were statistically significantly older than those in the migraine group. Therefore, we relied on statistical methods to adjust for 
potentially confounding effects, such as age (Table 3). Fifth, we attempted to consider the effect of confounding factors on the available data, but there were some contributing factors that we failed to assess (such as physical activity, education, smoking status, alcohol consumption, among others) and, therefore, we cannot not fully exclude the effects of all confounders on the study results.

\section{CONCLUSIONS}

The results of this large population-based observational study show that high dietary acid load intake had a positive association with odds of migraine onset. Thus, as a strategy to reduce dietary acid load, restricting the intake of acidogenic foods (such as meat, grains, and sugar that are high in protein and phosphorous) and those low in alkaline precursors may be considered a suggestion for reducing the odds migraine odds. However, due to preliminary nature of current evidence, caution is advised when assessing the results. Thus, further investigation with a large sample size in cohort studies and well-designed clinical trials are required to identify the exact association between dietary acid load and migraine clinical features or even related health outcomes.

\section{ACKNOWLEDGEMENTS}

We thank the participants of the present study. In particular, we extend our gratitude to Ms Jabbari and Sina University Hospital staff for their kind cooperation. The authors would also like to thank the epidemiology and biostatistics staff of the Research Development Centre of Sina Hospital for their technical assistance.

Funding. This research was supported by the Iranian Centre of Neurological Research, Neuroscience Institute, Grant Number 97-03-5439214. The funding role was in the design of the study; collection, analysis, and interpretation of data; and in writing the manuscript. No funding was received for the publication of this article.
Editorial Assistance. Editorial assistance for this article was provided by Ms.Carol Ashraf Eastwood Ahmdi (freelance) and funded by the authors. No financial support for this assistance was provided by the University.

Authorship. All authors meet the International Committee of Medical Journal Editors (ICMJE) criteria for authorship for this article. All authors had full access to the study data and take responsibility for the integrity of the data and the accuracy of the data analysis, and have given their approval for this version to be published.

Authors' Contributions. Mansoureh Togha and Soodeh Razeghi Jahromi jointly conceived and designed the study and contributed to the draft. Zeinab Ghorbani, Azita Hekmatdoost, Pegah Rafiee, Boshra Torkan, Pedram Shirani, Hossein Ansari, Ahmadreza Karami, Morvarid Nourmohammadi, Faezeh Khorsha, and Maryam Mousavi were involved in data gathering and performed statistical analysis of the research. Maryam Mousavi drafted the manuscript. All authors critically revised the manuscript for important intellectual contents. Mansoureh Togha gave the final approval of the version to be published. All authors have read and approved the manuscript.

Prior Presentation. This research has been previously presented at the 3rd International and the 15th Iranian Nutrition Congress on 19-21 December in 2018, by Shahid Beheshti University of Medical Sciences and Health Services, Iran Nutrition Association, Institute of Nutritional Research and Food Industries, Iranian Society of Nutrition in Tehran city. The current research was presented as a poster at this congress.

Disclosures. Maryam Mousavi, Soodeh Razeghi Jahromi, Mansoureh Togha, Zeinab Ghorbani, Azita Hekmatdoost, Pegah Rafiee, Boshra Torkan, Pedram Shirani, Hossein Ansari, Ahmadreza Karami, Morvarid Nourmohammadi, and Faezeh Khorsha personally had no financial, commercial, or academic conflicts of interest. 
Compliance with Ethics Guidelines. The research methods and study design were reviewed and approved by the ethics committee of the vice-chancellor of research affairs, Tehran University of Medical Sciences (Ethics code: IR.TUMS.NI.REC.1398.010). The study was conducted in accordance with the Helsinki Declaration of 1964 and its later amendments. Written informed consent was obtained from all studied subjects.

Data Availability. The data sets generated and/or analyzed during the current study are available from the corresponding author on reasonable request.

Open Access. This article is licensed under a Creative Commons Attribution-Non-Commercial 4.0 International License, which permits any non-commercial use, sharing, adaptation, distribution and reproduction in any medium or format, as long as you give appropriate credit to the original author(s) and the source, provide a link to the Creative Commons licence, and indicate if changes were made. The images or other third-party material in this article are included in the article's Creative Commons licence, unless indicated otherwise in a credit line to the material. If material is not included in the article's Creative Commons licence and your intended use is not permitted by statutory regulation or exceeds the permitted use, you will need to obtain permission directly from the copyright holder. To view a copy of this licence, visit https://creativecommons.org/licenses/by$\mathrm{nc} / 4.0 /$.

\section{REFERENCES}

1. Wijeratne T, Grisold W, Dodick D, Carroll W. World Brain Day 2019: migraine, the painful truth. Lancet Neurol. 2019;18(10):914.

2. Stovner LJ, Nichols E, Steiner TJ, et al. Global, regional, and national burden of migraine and tension-type headache, 1990-2016: a systematic analysis for the Global Burden of Disease Study 2016. Lancet Neurol. 2018;17(11):954-76.
3. Reuter U. GBD 2016: still no improvement in the burden of migraine. Lancet Neurol. 2018;17(11): 929-30.

4. Buse D, Manack A, Serrano D, Turkel C, Lipton RB. Sociodemographic and comorbidity profiles of chronic migraine and episodic migraine sufferers. J Neurol Neurosurg Psychiatry. 2010;81(4):428-32.

5. Martami F, Ghorbani Z, Abolhasani M, et al. Comorbidity of gastrointestinal disorders, migraine, and tension-type headache: a cross-sectional study in Iran. Neurol Sci. 2018;39(1):63-70.

6. Capuano A, De Corato A, Lisi L, Tringali G, Navarra $\mathrm{P}$, Russo CD. Proinflammatory-activated trigeminal satellite cells promote neuronal sensitization: relevance for migraine pathology. Mol Pain. 2009;5:43.

7. Dodick DW. A phase-by-phase review of migraine pathophysiology. Headache. 2018;58[Suppl 1]:4-16.

8. Arzani M, Jahromi SR, Ghorbani Z, et al. Gut-brain axis and migraine headache: a comprehensive review. J Headache Pain. 2020;21(1):1-12.

9. Ghorbani Z, Rafiee P, Fotouhi A, et al. The effects of vitamin $\mathrm{D}$ supplementation on interictal serum levels of calcitonin gene-related peptide (CGRP) in episodic migraine patients: post hoc analysis of a randomized double-blind placebo-controlled trial. J Headache Pain. 2020;21(1):1-13.

10. Fidan I, Yuksel S, Ymir T, Irkec C, Aksakal FN. The importance of cytokines, chemokines and nitric oxide in pathophysiology of migraine. J Neuroimmunol. 2006;171(1-2):184-8.

11. Ghorbani Z, Togha M, Rafiee P, et al. Vitamin D in migraine headache: a comprehensive review on literature. Neurol Sci. 2019;40(12):2459-77.

12. Bowen EJ, Schmidt TW, Firm CS, Russo AF, Durham PL. Tumor necrosis factor-alpha stimulation of calcitonin gene-related peptide expression and secretion from rat trigeminal ganglion neurons. J Neurochem. 2006;96(1):65-77.

13. Yücel M, Kotan D, Gurol Çiftçi G, Çiftçi I, Cikriklar H. Serum levels of endocan, claudin-5 and cytokines in migraine. Eur Rev Med Pharmacol Sci. 2016;20(5):930-6.

14. Salehi H, Aminianfar M, Ranjbar-Naeeni A, Saidi A, Rastgoo F. Comparison of serum CRP in migraine sufferers and normal population. 2013;16(1):13-6.

15. Martami F, Jahromi SR, Togha M, Ghorbani Z, Seifishahpar M, Saidpour A. The serum level of inflammatory markers in chronic and episodic migraine: a case-control study. Neurol Sci. 2018;39(10):1741-9. 
16. Jahromi SR, Ghorbani Z, Martelletti P, Lampl C, Togha M. Association of diet and headache. J Headache Pain. 2019;20(1):1-11.

17. Pedoto A, Nandi J, Oler A, Camporesi EM, Hakim TS, Levine RA. Role of nitric oxide in acidosis-induced intestinal injury in anesthetized rats. J Lab Clin Med. 2001;138(4):270-6.

18. Kellum JA, Song M, Almasri E. Hyperchloremic acidosis increases circulating inflammatory molecules in experimental sepsis. Chest. 2006;130(4): 962-7.

19. Wu T, Seaver P, Lemus H, Hollenbach K, Wang E, Pierce JP. Associations between dietary acid load and biomarkers of inflammation and hyperglycemia in breast cancer survivors. Nutrients. 2019;11(8):1913.

20. Akter S, Eguchi M, Kurotani K, et al. High dietary acid load is associated with increased prevalence of hypertension: the Furukawa Nutrition and Health Study. Nutrition. 2015;31(2):298-303.

21. Farhangi MA, Nikniaz L, Nikniaz Z. Higher dietary acid load potentially increases serum triglyceride and obesity prevalence in adults: An updated systematic review and meta-analysis. PloS ONE. 2019;14(5):e0216547.

22. Carnauba RA, Baptistella AB, Paschoal V, Hübscher GH. Diet-induced low-grade metabolic acidosis and clinical outcomes: a review. Nutrients. 2017;9(6): 538.

23. Adeva MM, Souto G. Diet-induced metabolic acidosis. Clin Nutr. 2011;30(4):416-21.

24. Hajjarzadeh S, Mahdavi R, Shalilahmadi D, Nikniaz Z. The association of dietary patterns with migraine attack frequency in migrainous women. Nutr Neurosci. 2020;23(9):724-30.

25. Frassetto LA, Todd KM, Morris RC Jr, Sebastian A. Estimation of net endogenous noncarbonic acid production in humans from diet potassium and protein contents. Am J Clin Nutr. 1998;68(3): 576-83.

26. Jahromi SR, Togha M, Ghorbani Z, et al. The association between dietary tryptophan intake and migraine. Neurol Sci. 2019;40(11):2349-55.

27. Huang T-C, Wang S-J. The International Classification of Headache Disorders, 3rd edn (beta version). Cephalalgia. 2013;33(9):629-808. https://doi.org/ $10.1177 / 0333102413485658$.

28. Esfahani FH, Asghari G, Mirmiran P, Azizi F. Reproducibility and relative validity of food group intake in a food frequency questionnaire developed for the Tehran Lipid and Glucose Study. J Epidemiol. 2010;20(2):150-8.

29. Ghafarpour M H-RA, Kianfar $H$. The manual for household measures, cooking yields factors and edible portion of food. Tehran: Nashre Olume Keshavarzy; 1999.

30. Azar M, Sarkisian E. Food composition table of Iran. Tehran: national nutrition and food research institute: Shahid Beheshti University; 1980, p 65.

31. Food and Nutrition Information Center, US Department of Agriculture. Food composition table (FCT); 2009. http://www.nal.usda.gov/fnic/ foodcomp.

32. Remer T, Dimitriou T, Manz F. Dietary potential renal acid load and renal net acid excretion in healthy, free-living children and adolescents. Am J Clin Nutr. 2003;77(5):1255-60.

33. Zwart SR, Hargens AR, Smith SM. The ratio of animal protein intake to potassium intake is a predictor of bone resorption in space flight analogues and in ambulatory subjects. Am J Clin Nutr. 2004;80(4): 1058-65.

34. Moghadam SK, Bahadoran Z, Mirmiran P, Tohidi M, Azizi F. Association between dietary acid load and insulin resistance: Tehran Lipid and Glucose Study. Prevent Nutr Food Sci. 2016;21(2):104.

35. Murakami K, Livingstone MBE, Okubo H, Sasaki S. Higher dietary acid load is weakly associated with higher adiposity measures and blood pressure in Japanese adults: The National Health and Nutrition Survey. Nutr Res. 2017;44:67-75.

36. Ariyanfar S, Jahromi SR, Rezaeimanesh N, et al. Fruit and vegetable intake and odds of pediatric migraine. Nutr Food Sci. 2019;50(5):829-40.

37. Nazari F, Eghbali M. Migraine and its relationship with dietary habits in women. Iran J Nurs Midwif Res. 2012;17(2 Suppl1):S65.

38. Evans EW, Lipton RB, Peterlin BL, Ret al. Dietary intake patterns and diet quality in a nationally representative sample of women with and without severe headache or migraine. Headache. 2015;55(4):550-61.

39. Altunkaynak Y, Ozturk M, Ertem DH, et al. Serum lactic acid and pyruvic acid levels in patients with migraine and tension type headache. Dusunen Adam. 2013;26(3):276.

40. Okada H, Araga S, Takeshima T, Nakashima K. Plasma lactic acid and pyruvic acid levels in migraine and tension-type headache. Headache. 1998;38(1):39-42. 
41. Pedoto A, Caruso JE, Nandi J, et al. Acidosis stimulates nitric oxide production and lung damage in rats. Am J Respir Crit Care Med. 1999;159(2): 397-402.

42. Olesen J, Thomsen LL, Iversen H. Nitric oxide is a key molecule in migraine and other vascular headaches. Trends Pharmacol Sci. 1994;15(5): 149-53.

43. Bowen EJ, Schmidt TW, Firm CS, Russo AF, Durham PL. Tumor necrosis factor- $\alpha$ stimulation of calcitonin gene-related peptide expression and secretion from rat trigeminal ganglion neurons. J Neurochem. 2006;96(1):65-77.

44. DeBustros A, Baylin SB, Levine M, Nelkin BD. Cyclic AMP and phorbol esters separately induce growth inhibition, calcitonin secretion, and calcitonin gene transcription in cultured human medullary thyroid carcinoma. J Biol Chem. 1986;261(17): 8036-41.

45. Tverberg L, Russo A. Regulation of the calcitonin/calcitonin gene-related peptide gene by cellspecific synergy between helix-loop-helix and octamer-binding transcription factors. J Biol Chem. 1993;268(21):15965-73.

46. Covelli V, Munno I, Pellegrino N, et al. In vivo administration of propranolol decreases exaggerated amounts of serum TNF-alpha in patients with migraine without aura. Pos Mech Act Acta Neurol. 1992;14(4-6):313-9.

47. Kopp S. Neuroendocrine, immune, and local responses related to temporomandibular disorders. J Orofacial Pain. 2001;15(1):9-28.
48. Leistad RB, Stovner LJ, White LR, Nilsen KB, Westgaard RH, Sand T. Noradrenaline and cortisol changes in response to low-grade cognitive stress differ in migraine and tension-type headache. J Headache Pain. 2007;8(3):157.

49. Jahromi SR, Abolhasani M, Ghorbani Z, et al. Bariatric surgery promising in migraine control: a controlled trial on weight loss and its effect on migraine headache. Obes Surg. 2018;28(1):87-96.

50. Agostoni E, Aliprandi A. Migraine and hypertension. Neurol Sci. 2008;29(1):37.

51. Gardener H, Monteith T, Rundek T, Wright CB, Elkind MS, Sacco RL. Hypertension and migraine in the Northern Manhattan Study. Ethn Dis. 2016;26(3):323.

52. David LA, Maurice CF, Carmody RN, et al. Diet rapidly and reproducibly alters the human gut microbiome. Nature. 2014;505(7484):559-63.

53. Yu-Jie Dai M, Hai-Yan Wang M, Xi-Jian Wang M, Alan David Kaye M. Potential beneficial effects of probiotics on human migraine headache: a literature review. Pain Phys. 2017;20:E251-E5.

54. Martami F, Togha M, Seifishahpar M, et al. The effects of a multispecies probiotic supplement on inflammatory markers and episodic and chronic migraine characteristics: a randomized doubleblind controlled trial. Cephalalgia. 2019;39(7): 841-53.

55. Vetvik KG, MacGregor EA. Sex differences in the epidemiology, clinical features, and pathophysiology of migraine. Lancet Neurol. 2017;16(1):76-87. 